as well as a series of work of exploring the best practices for prevention and treatment of syphilis at the present stage, including improving syphilis surveillance system, diagnosis and treatment, developing the demonstration sites for syphilis control policies implementation, spurring innovation practices for expanding HIV/syphilis screen test among high risk groups.

\section{Plenary Session PL03}

\section{Tuesday 15 September 2015}

\subsection{5am - 10.45am}

\section{PL03.1 ADVANCES IN CHLAMYDIA GENETICS - FROM UNDERSTANDING BASIC BIOLOGY TO VACCINE DESIGN}

Raphael Valdivia. Duke University Medical Center, Durham, USA

10.1136/sextrans-2015-052270.8

Chlamydia trachomatis is the most widely disseminated bacterial sexually transmitted infection and a leading cause of pelvic inflammatory disease and infertility. The last five years have witnessed a technological revolution in the analysis and characterisation of the function of chlamydia virulence factors and how they modulate host cellular functions. As a result, we are gaining unprecedented insights into the mechanisms underlying chlamydia-mediated inflammation and tissue damage as well as learning how chlamydia adapts to the human host environment. Prominent among these new technologies are methods to perform genetic and molecular genetic analysis in these bacteria, which until very recently were considered to be "genetically intractable". I will review some of the key developments in the emerging field of chlamydia genetics and provide examples of how these approaches are leading to the discovery of new virulence factors and strategies used by this pathogen to modulate innate immune responses. Finally, I will discuss recent progress in how these molecular genetic approaches are being used for the design of new vaccines and to better understand adaptive and innate immune responses to chlamydia infections.

\section{PL03.2 SCREENING FOR CHLAMYDIA: DOES IT WORK, RESULTS FROM ACCEPt}

Jane Hocking. Professor of Epidemiology, Centre for Women's Health, Gender and Society, University of Melbourne, Melbourne, Australia

\subsection{6/sextrans-2015-052270.9}

Chlamydia trachomatis is the most commonly reported bacterial sexually transmitted infection and its greatest burden is among young adults with community-based studies reporting prevalence estimates of 3 to 5\% among 16 to 29 year olds in Australia. As over $80 \%$ of infections are asymptomatic, screening is the main way to detect cases. However, there is considerable debate about the effectiveness of chlamydia screening. In response to this concern, the Australian Government funded the Australian Chlamydia Control Effectiveness Pilot (ACCEPt) to assess the feasibility, acceptability, efficacy and cost-effectiveness of annual chlamydia testing for sexually active 16 to 29 year old men and women attending general practice.
ACCEPt is being evaluated using a cluster randomised controlled trial design. A total of 143 general practice clinics in 52 geographical areas (clusters) are participating. An intervention to support increased chlamydia testing, including incentive payments for each test done, quarterly feedback on testing performance and computer prompts, has been allocated at the cluster level and all general practice clinics within each cluster are participating. The primary outcome of ACCEPt is change in chlamydia prevalence among 16 to 29 -year-old men and women attending general practice. This will be assessed as the difference in chlamydia prevalence between a baseline prevalence survey conducted among a consecutive sample of 16- to 29-year-old men and women attending all participating clinics prior to randomisation and a follow-up survey conducted at the conclusion of the trial. The fundamental premise of this trial is that increased levels of testing can be achieved by providing support to clinics, and that once levels of chlamydia testing are sufficiently increased, the prevalence of chlamydia will fall. Preliminary results from ACCEPt will be presented.

\section{Plenary Session PL04}

\section{Tuesday 15 September 2015}

\section{$4.00 \mathrm{pm}-5.30 \mathrm{pm}$}

\section{PL04.1 MALE CIRCUMCISION FOR STI PREVENTION: HOW WELL DOES IT WORK AND HOW IS IT DONE?}

Kawango Agot. University of Nairobi and CEO, Impact Research \& Development Organization, Kenya

\subsection{6/sextrans-2015-052270.10}

Interest in the possible association between male circumcision (MC) and sexually transmitted infections (STIs), including HIV, began in earnest in 1980s with studies exploring the tripartite relationship of circumcision, genital ulcer disease and HIV. The interest gathered momentum in the 1990s with more studies exploring these associations beyond within and Africa. In 2002, the first randomised controlled trial (RCT) was initiated in Kisumu, Kenya, with two others following shortly after in Orange Farm, South Africa and Rakai, Uganda. Although the RCTs focused primarily on MC and HIV, they also explored associations of MC and different STIs. This presentation focuses on the association between $\mathrm{MC}$ and human papillomavirus (HPV), herpes simplex virus type 2 (HSV-2), syphilis, Neisseria gonorrhoeae, Chlamydia trachomatis and Trichomonas vaginalis reported by the three RCTs as well as in systematic reviews, meta-analyses and other studies that followed.

The protective effect of MC against HPV was consistent across the three trials; in addition, the Uganda trial found that female partners of circumcised men had lower rates of HPV infection. While results from the RCTs were somewhat inconsistent on the association between MC and gonorrhoeae, HSV-2, syphilis, chlamydia and trichomoniasis, several meta-analyses and post-RCT studies have reported that MC protects against most of these conditions. As a result of these benefits, several MC devices have been developed to fast-track the provision of MC services, notably PrePex and ShangRing that have been preapproved by the World Health Organization. 